\title{
Screening of FDA Approved Drugs Against SARS-CoV-2 Main Protease: Coronavirus Disease
}

\author{
Vijayakumar Balakrishnan ${ }^{1}$ [D $\cdot$ Karthik Lakshminarayanan ${ }^{2}$
}

Accepted: 18 September 2020 / Published online: 28 September 2020

(c) Springer Nature B.V. 2020

\begin{abstract}
At the end of December 2019, a new strain of coronavirus was identified in the Wuhan city of Hubei province in China. Within a shorter period of time, an unprecedented outbreak of this strain was witnessed over the entire Wuhan city. This novel coronavirus strain was later officially renamed as COVID-19 (Coronavirus disease 2019) by the World Health Organization. The mode of transmission was human-to-human contact and hence resulted in a rapid surge across the globe where more than 24 million people have been infected with COVID-19. In the current scenario, finding potent drug candidates for the treatment of COVID-19 has emerged as the most challenging task for clinicians and researchers worldwide. Identification of new drugs and vaccine development may take from a few months to years based on the clinical trial processes. To overcome the several limitations involved in identifying and bringing out potent drug candidates for treating COVID-19, in the present study attempts were made to screen the FDA approved drugs using High Throughput Virtual Screening (HTVS). The COVID-19 main protease (COVID-19 Mpro) was chosen as the drug target for which the FDA approved drugs were initially screened with HTVS. The drug candidates that exhibited favorable docking score, energy, and emodel calculations were further taken for performing Induced Fit Docking (IFD) using Schrodinger's GLIDE. From the flexible docking results, the following four FDA approved drugs Sincalide, Pentagastrin, Ritonavir, and Phytonadione were identified. In particular, Sincalide and Pentagastrin can be considered potential key players for the treatment of COVID-19 disease.
\end{abstract}

Keywords Coronavirus $\cdot$ COVID-19 $\cdot$ FDA approved drugs $\cdot$ High throughput virtual screening $\cdot$ Sincalide $\cdot$ Pentagastrin

\section{Introduction}

A new strain of Coronavirus(s) $(\mathrm{CoVs})$ was identified in the concluding weeks of December 2019 and emerged in an alarming outbreak in the Wuhan city of Hubei province in China (Lee and Hsueh 2020). Initially the World Health Organization (WHO) has named this strain as 2019-nCoV and officially declared the outbreak of this virus in China on January 30, 2020 (Rodríguez-Morales et al. 2020). Following the consultations with the World Organization for Animal Health and the Food and Agriculture Organization of the United Nations, later on, WHO officially renamed

Vijayakumar Balakrishnan

vijaybioscience@ifsc.usp.br; vijaybioscience@gmail.com

1 São Carlos Institute of Physics (IFSC), University of São Paulo, Av. Joao Dagnone, 1100 - Jardim Santa Angelina, São Carlos 13563-120, Brazil

2 ToxiVen Biotech, Siva Nagar, Kovaipudur, Tamil Nadu 641042, India this strain as COVID-19 on February 11, 2020. Since the mode of transmission was found to be human-to-human contact, by the end of February 2020, over 90,000 people were infected with COVID-19 among which as many as 3000 cases have succumbed to this deadly infection. This rapid surge in people being infected with COVID-19 has forced the WHO to declare this a Global Public Health Emergency on 4th March 2020.

Belongs to the Nidovirales order, CoVs genome is comprised of a linear and positive-sense RNA molecule (Kim et al. 2020; Zhou et al. 2020). The Coronaviridae family is further classified into four genera: Alpha, Beta, Delta and Gamma CoVs. Members of these families generally consist of three to four envelope proteins with the membrane protein being the most abundant one. As of date, seven coronaviruses have been identified that infect humans. The strains of HCoV-OC43 and HCoV-HKU1 from Betacoronavirus and HCoV-229E from Alphacoronavirus were found to be the causative agents for common colds and severe lower respiratory tract infections across all the age groups (Falsey 
et al. 1997; Graat et al. 2003; Mackay et al. 2004; van Elden et al. 2004; Vabret et al. 2003; Vabret et al. 2008). The pandemic COVID-19 strain was initially considered to be related to the Beta coronaviruses strain of SARS-CoV (2002 outbreak) and MERS-CoV (2012 outbreak). Later on, it was further concluded that COVID-19 is closely related to SARS-CoV and genetically clusters within Beta coronavirus subgenus Sarbecovirus (Zhou et al. 2020).

As of date (August 29, 2020), the total number of confirmed cases have reached up to 24.9 million worldwide among which about 841,832 deaths have been reported for this viral infection. Among the total cases, around 6.7 million were found to be active with 17.3 million people were officially declared as recovered fully from the infection. In the height of this crisis, the rate and scale of COVID19 spreading have been considered to be far worse when compared to that of the previous coronaviral epidemics. Moreover, due to the lack of investigations pertaining to the treatment of COVID-19, no specific therapies have been identified for treating this dreadful infection (RodríguezMorales et al. 2020). A preliminary study using the combination of protease inhibitor lopinavir/ritonavir has been reported for the treatment of COVID-19 infected patients (Lu, 2020). This combination has been generally used for treating patients affected by human immunodeficiency virus (HIV). Several existing antiviral agents such as nucleoside analogues, neuraminidase inhibitors, tenofovir disoproxil (TDF) and lamivudine (3TC) have also been tested against pathogenic CoVs (Lu 2020). The main protease(Mpro)/chymotrypsin-like protease (3CLpro) and RNA polymerase of COVID-19 are considered attractive drug targets for designing potent antiviral compounds. A recent study indicated that among the tested compounds Nelfinavir was identified as the best potential inhibitor against COVID-19 Mpro (Zhijian et al. 2020). Similarly, another study suggested that the nucleoside analogue Remdesivir was found effective against COVID-19 Mpro (Chang et al. 2020). However, as these findings remain unapproved, results from these preliminary studies cannot be applied for therapeutic use and in the subsequent clinical setting for the treatment of COVID-19-infected patients (Lu 2020; Michelle et al. 2020).

In order to overcome the limitations prevailing in identifying potent antiviral drugs, the present study describes a combined molecular modeling approach carried out against COVID-19 Mpro. For this, the complete list of FDA approved drugs ( 1500 compounds) were retrieved from the Binding Database (BindingDB). Using the HTVS, drugs with favorable docking score, glide energy, glide emodel were further subjected to Induced Fit Docking (IFD) using Schrodinger's GLIDE. Finally, all the chosen drugs used in the IFD studies were analyzed for the favorable docking score, glide energy, glide emodel and interaction studies (hydrogen bond and hydrophobic) with COVID-19 Mpro.
The following four drugs Sincalide, Pentagastrin, Ritonavir and Phytonadione have exhibited better results with COVID19 Mpro. In particular, the potent inhibition of Sincalide and Pentagastrin drugs along with similar structurally related inhibitors could be used as a key player in the treatment of COVID-19 disease. The findings of the present study will provide other doctors/researchers with opportunities to treat the patients or identify the right drug to combat COVID-19.

\section{Methodology}

Glide software (v11.1, Schrödinger, 2017, USA) was used for the molecular modeling calculations involving rigid (High Throughput Virtual screening) and flexible (Induced Fit Docking) docking studies. Chimera (Pettersen et al. 2004) and glide ligand interaction display software were used for graphical visualization, analysis of the hydrogen bond interaction and hydrophobic interactions between the active site amino acids and FDA drugs.

\section{Protein Preparation}

The crystal structure of COVID-19 Mpro (PDB: 6LU7) in complex with N3 inhibitor (Fig. 1) was retrieved from PDB (www.rcsb.org). The protein comprises of 3 domains viz., domain I (residues 8-101), II (residues 102-184) and III (residues 201-303). The N3 inhibitor maintained the highest numbers of $\mathrm{H}$-Bond interactions with domain II (active site residues) (Jin et al. 2020). The raw complex structure retrieved from PDB was not suitable for the screening of FDA approved drugs using HTVs and IFD molecular modeling studies. In this connection, protein preparation (Schrödinger modules) was employed to fix the missed out information of bond orders, topologies, formal atomic charges, ionization, tautomeric states and removed unwanted water molecules in the structure. Moreover, non-hydrogen atoms were energy minimized until the average root mean square deviation reaches $0.3 \AA$ and the amino acid flips were assigned.

\section{Drug Selection}

FDA-approved drugs ( 1500 compounds) were recovered from the BindingDB with 3D conformations (https://www. bindingdb.org/bind/ByFDAdrugs.jsp). All these drugs were clustered within different categories such as peptide drugs, anti-HIV, anti-malarial, anti-Hepatitis $\mathrm{C}$ virus, anti-bacterial, anti-fungal, anti-inflammatory, natural source drugs and various other categories. In addition to this, all these drugs have also contained reliable molecular information with added hydrogens, 3D conversion, realistic bond lengths and bond angles, low energy structure with correct chirality's, 


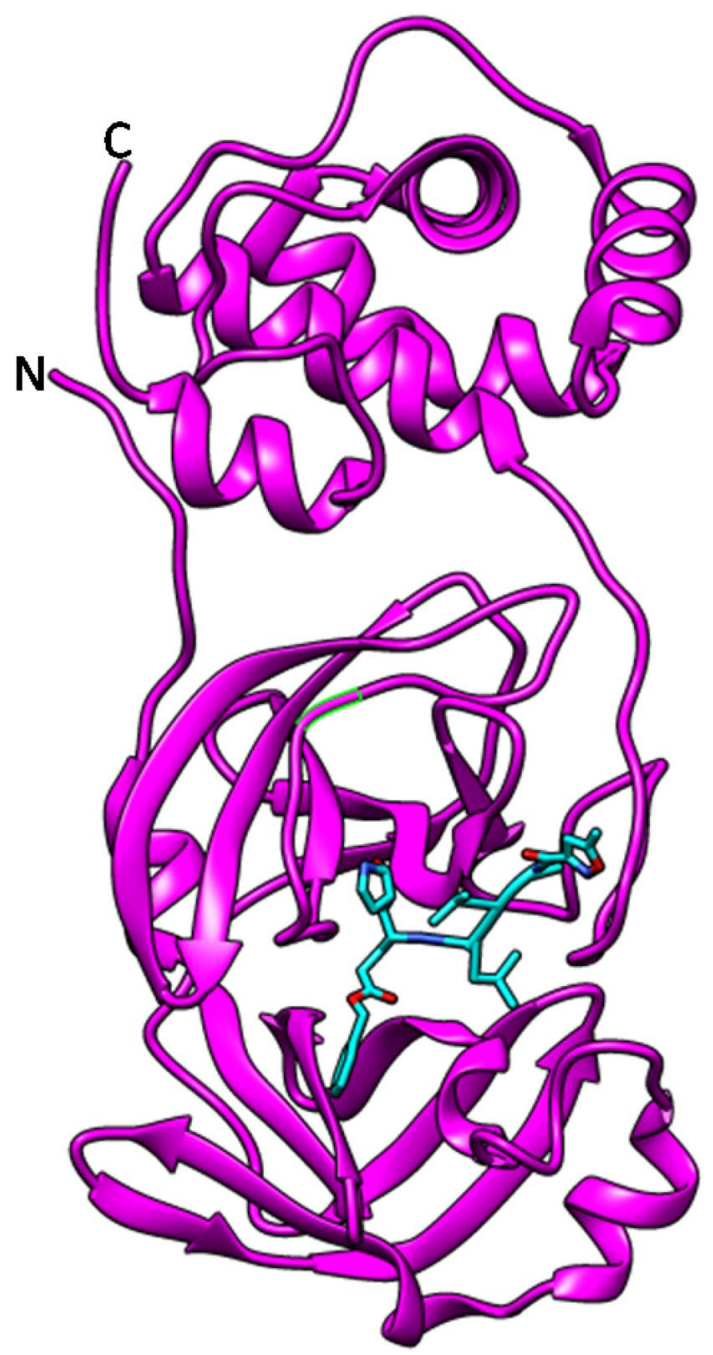

Fig. 1 Crystal structure of COVID-19 Mpro in complex with N3 inhibitor (6LU7)

ionization states, tautomers, stereochemistries and ring conformation.

\section{High Throughput Virtual Screening (HTVs)}

HTVs is one of the methods used for screening multiple compounds against active site key residues in the previously calculated grid receptor. The energy minimized complex of COVID-19 Mpro with the N3 inhibitor was loaded in the workspace. Following this, the receptor grid generation process was employed where the active site of the receptor was calculated automatically by picking the N3 inhibitor. This process of docking the drug at the grid box (active site) yields a more accurate scoring function and energy of the protein-ligand complex. The docking score, glide energy, glide emodel and non-bonded interactions were analyzed in the HTVs results.

\section{Induced Fit Docking Studies}

In IFD (flexible docking), the proteins undergo side-chain or backbone conformation or both in many cases when a drug binds at the active site cavity. These conformational changes are one of the key processes to predict concomitant structural movements and accurate drug binding in the active site of the receptor. The best 4 drugs chosen from the HTVs results were further subjected to IFD studies. These drugs were redocked at the active site of the target. Docking score, glide energy, and glide emodel were obtained from the energetically favorable docked complexes and the best poses were chosen for analyzing the hydrogen bond and hydrophobic interactions.

\section{Results and Discussion}

\section{High Throughput Virtual Screening (HTVs)}

FDA-approved drugs that comprises peptide-based drugs, anti-HIV, anti-malarial, anti-Hepatitis $\mathrm{C}$ virus, anti-bacterial, anti-fungal, anti-inflammatory, drugs of natural sources and various other categories were screened against COVID-19 Mpro. From the results, it was observed that few peptide-based and antiviral drugs along with natural source drugs exhibited maximum glide score, energy and emodel (Table 1). The screened drugs were also analyzed for the non-bonded interactions with the active site residues and following this the chosen drugs were subjected for further IFD studies.

\section{Anti-HIV Drugs}

Anti-HIV drugs of Ritonavir, Indinavir and Lopinavir were redocked at the active site of the target using HTVs (Table 1). Based on the HTVS results, among the three drugs, Ritonavir (Fig. 2a) alone was taken for further IFD studies. The IFD complex of Ritonavir has exhibited a docking score -11.292 , glide energy -81.842 and glide emodel - 128.743 (Fig. 3a; Table 2). The drug also maintained nonbonded interactions with the active site residues.

\section{Peptide Drugs}

Sincalide/cholecystokinin (CCK-8) is a C-terminal octapeptide that is naturally occurring in gastrointestinal hormone (Fig. 2b). The Sincalide drug was bound well at the active site of COVID-19 Mpro. This IFD complex has possessed docking score -14.183 , glide energy -114.183 , 
Table 1 Docking score, glide energy and glide emodel in SP docked complexes

\begin{tabular}{llllllll}
\hline FDA drug & Docking score & $\begin{array}{l}\text { Glide energy } \\
(\mathrm{Kcal} / \mathrm{mol})\end{array}$ & $\begin{array}{l}\text { Glide emodel } \\
(\mathrm{Kcal} / \mathrm{mol})\end{array}$ & FDA drug & Docking score & $\begin{array}{l}\text { Glide energy } \\
(\mathrm{Kcal} / \mathrm{mol})\end{array}$ & $\begin{array}{l}\text { Glide emodel } \\
(\mathrm{Kcal} / \mathrm{mol})\end{array}$ \\
\hline N3* & -11.27 & -80.96 & -125.63 & Ciprofloxacin & -7.628 & -43.969 & -63.051 \\
Sincalide & -12.09 & -93.783 & -152.565 & Indomethacin & -7.653 & -49.226 & -71.042 \\
Pentagastrin & -11.225 & -83.516 & -141.213 & Telaprevir & -8.29 & -63.439 & -92.735 \\
Ritonavir & -11.644 & -81.385 & -131.095 & Boceprevir & -6.181 & -50.987 & -65.677 \\
Indinavir & -8.409 & -58.818 & -80.42 & Fluconazole & -7.859 & -46.243 & -66.068 \\
Lopinavir & -8.148 & -63.668 & -89.424 & Miconazole & -6.999 & -50.924 & -68.483 \\
Mefloquine & -7.454 & -41.733 & -56.069 & Dicumarol & -6.202 & -40.023 & -52.402 \\
Amodiaquine & -6.434 & -43.429 & -59.249 & Phytonadione & -5.313 & -43.995 & -55.586 \\
\hline
\end{tabular}

*Clinical trial

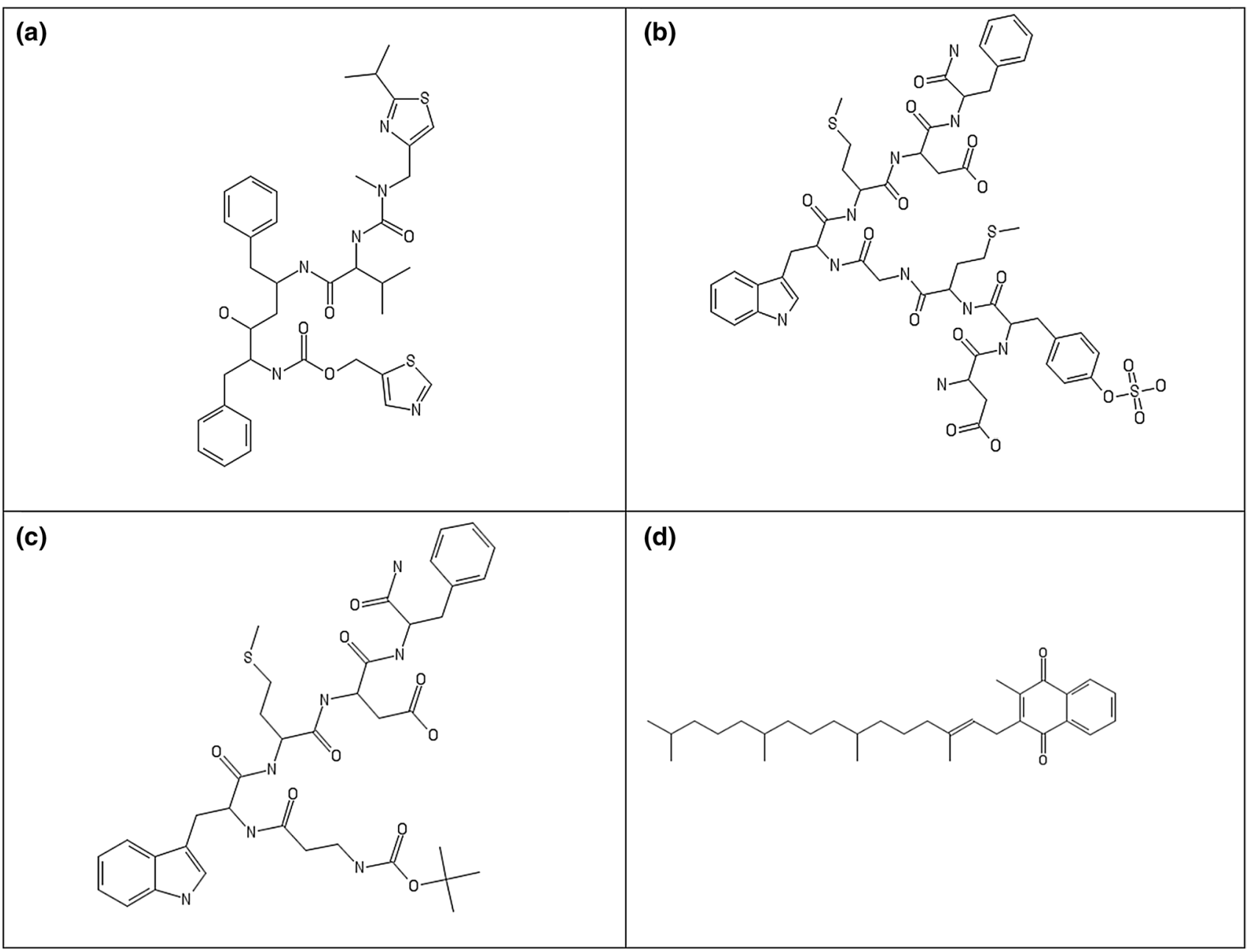

Fig. 2 2-D Chemical diagrams of a Ritonavir, b Sincalide, $\mathbf{c}$ Pentagastrin and $\mathbf{d}$ Phytonadione (Vitamine $\mathrm{K}_{1}$ )

and glide emodel -220.008 (Fig. 3b; Table 2) and has very good non-bonded interactions with the active site residues. It has been well established that the Potato protease inhibitors increases the circulation of cholecystokinin levels through a trypsin-dependent mechanism (Komarnytsky et al. 2011).

Pentagastrin is a synthetic polypeptide (Fig. 2c) that was bound at the active site of COVID-19 Mpro. The IFD 
(a)

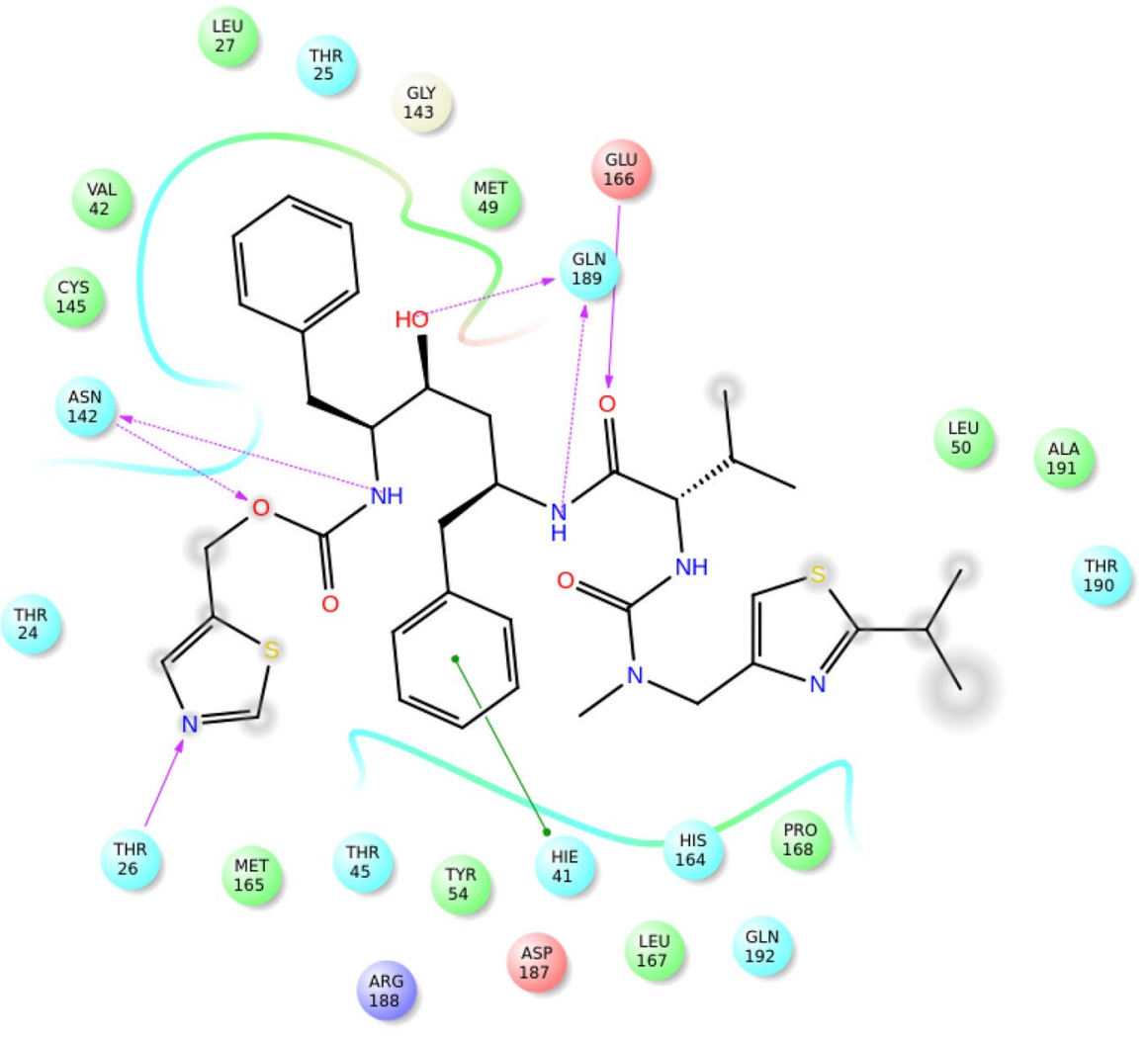

(b)

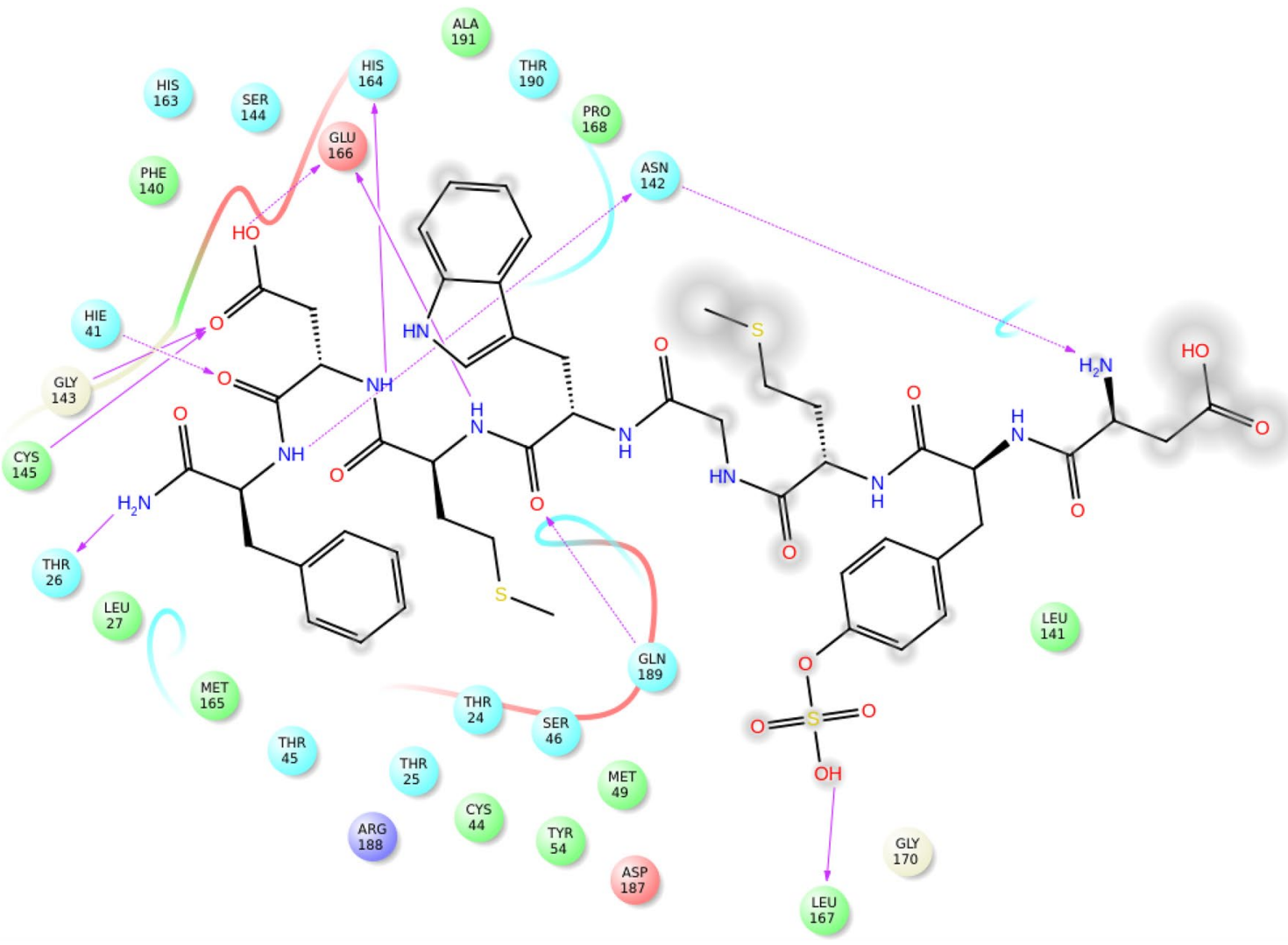

Fig. 3 Hydrogen bond interactions of a Ritonavir, b Sincalide, $\mathbf{c}$ Pentagastrin and d Phytonadione with active site residues of SARS-CoV-2 Main Protease 
(c)

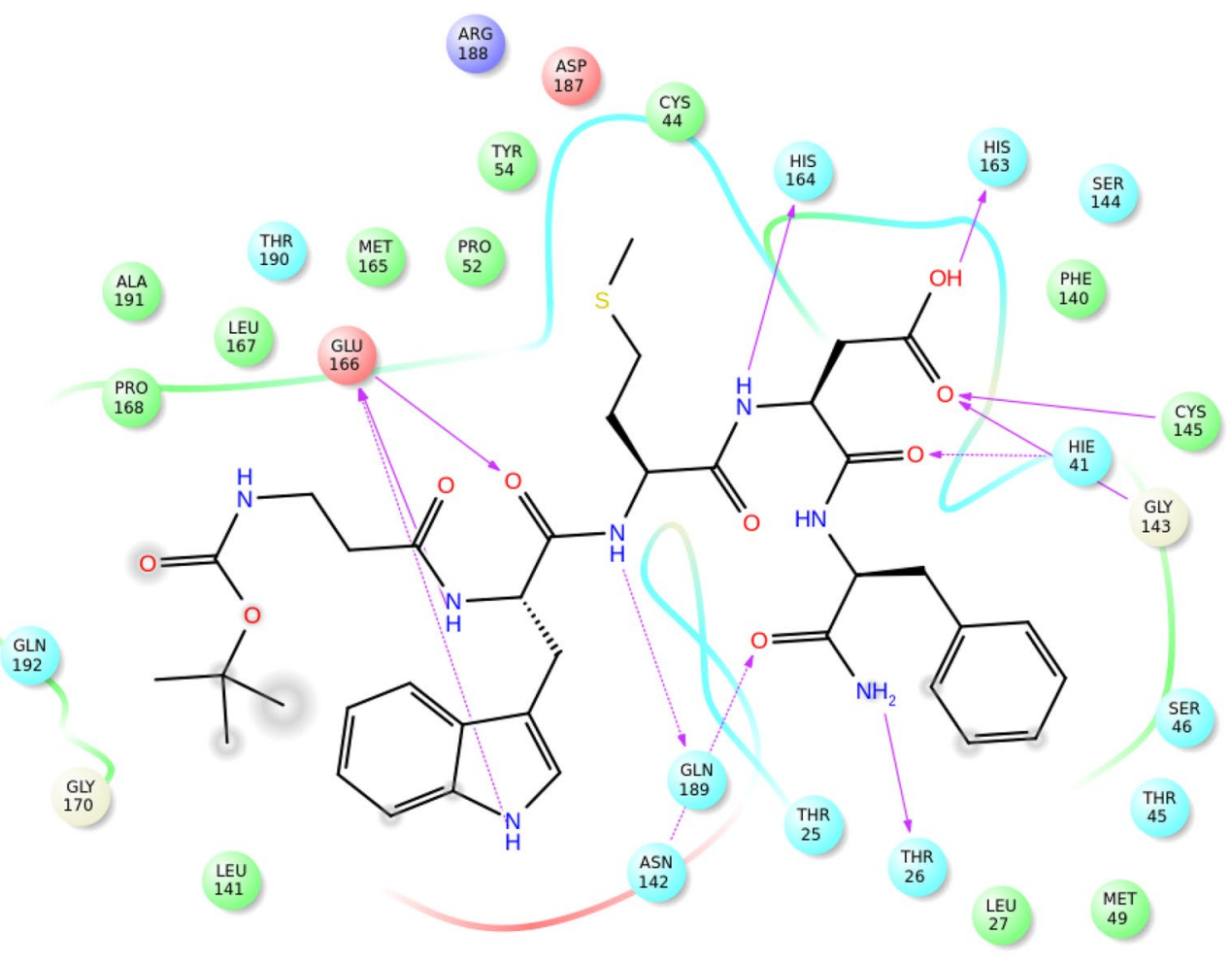

(d)

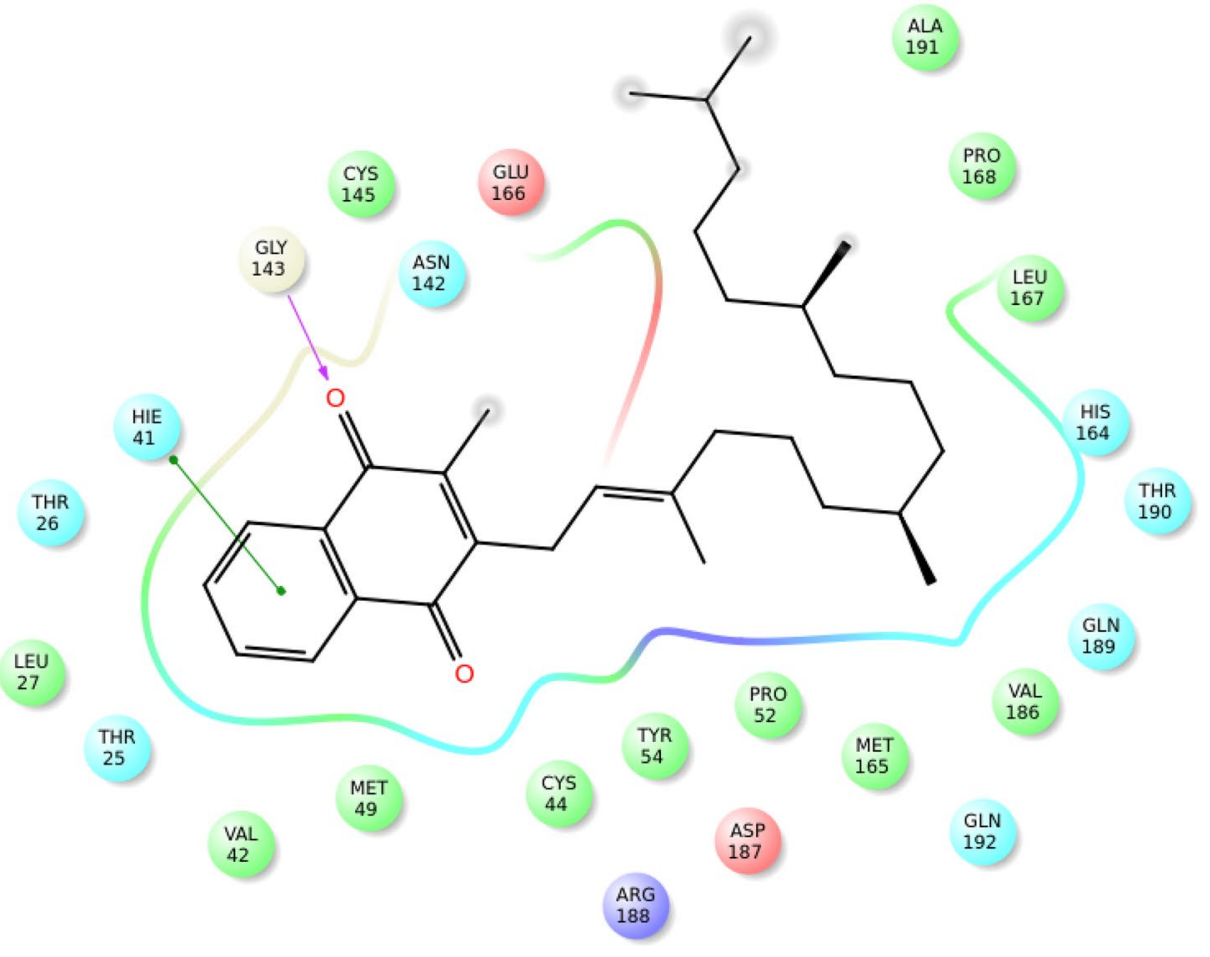

Fig. 3 (continued) 
Table 2 Docking score, glide energy and glide emodel in Induced fit docked complexes

\begin{tabular}{lccc}
\hline FDA drug & Docking score & $\begin{array}{l}\text { Glide energy } \\
(\mathrm{Kcal} / \mathrm{mol})\end{array}$ & $\begin{array}{l}\text { Glide emodel } \\
(\mathrm{Kcal} / \mathrm{mol})\end{array}$ \\
\hline N3* & -13.145 & -88.302 & -152.882 \\
Sincalide & -14.183 & -114.183 & -220.008 \\
Pentagastrin & -15.316 & -103.681 & -195.461 \\
Ritonavir & -11.292 & -81.842 & -128.743 \\
Phytonadione & -9.578 & -57.931 & -84.813 \\
\hline
\end{tabular}

*Clinical trial

complex possessed a docking score -15.316 , glide energy -103.681, and glide emodel - 195.461 (Fig. 3c; Table 2). In addition, the peptide also exhibits very good non-bonded interactions with the active site residues.

\section{Natural Source Drugs (Vitamin K)}

Dicoumarol and Phytonadione (Vitamin K1) are vitamins. These drugs were redocked at the active site of the target in HTVs (Table 1). The IFD complex of Phytonadione (Fig. 2d) has possessed a docking score -9.578 , glide energy -57.931 and glide emodel -84.813 (Fig. 3d; Table 2). The drug maintained non-bonded interactions with the active site residues. Phytonadione is rich in Green leafy vegetables. Medical expertise can suggest this nutritional diet for COVID-19 affected patients based on further reconfirmation.

\section{Conclusions}

In this study, the FDA-approved ( 1500 compounds) drugs were screened using HTVs. Based on the results, four drugs were compared with the N3 inhibitor which was previously redocked at the active site of COVID-19 Mpro. The docking score, glide energy, glide emodel, hydrogen bond and hydrophobic interactions were analyzed. The four drugs Sincalide, Pentagastrin, Ritonavir and Phytonadione have been found to possess better results with COVID-19 Mpro (Coronavirus disease). In particular, the potent inhibition exhibited by the FDA approved Sincalide and Pentagastrin drugs were found to be more efficient in terms of docking score, energy and capability of forming hydrogen bond interactions with the active site residues. Altogether, the FDA approved drugs identified in the present study can be utilized in combinatorial therapy where these drugs, as well as inhibitors structurally similar to these peptide drugs, can be used in combination for the treatment of COVID-19 disease.
Acknowledgements This work was supported by the National Institute of Science and Technology -Biorational Control of Insects and Pests (INCT-CBIP; Grant Numbers FAPESP 2008/51859-5 and CNPq 573742/2008-1), São Paulo State Foundation for Research (FAPESP; Grant Numbers 2018/00492-4. The authors would also like to thank Prof. Dr. Andre Luis Berteli Ambrosio and Prof. Dr. Rafael Victório Carvalho Guido for the research support and software facility.

\section{Compliance with Ethical Standards}

Conflict of interest The authors declare that they have no conflict of interest.

\section{References}

Chang Y, Tung Y, Lee K, Chen T, Hsiao Y, Chang H, Hsieh T, Su C, Wang S, Yu J, Shih S, Lin Y, Lin Y, Tu YE, Hsu C, Juan H, Tung C, Chen C (2020) Potential therapeutic agents for COVID-19 based on the analysis of protease and RNA polymerase docking. Preprints 2020020242 https://doi.org/10.20944/preprints202002 .0242.v2)

Falsey AR, McCann RM, Hall WJ, Criddle MM, Formica MA, Wycoff D (1997) The "common cold" in frail older persons: impact of rhinovirus and coronavirus in a senior daycare center. J Am Geriatr Soc 45:706-711

Graat JM, Schouten EG, Heijnen ML, Kok FJ, Pallast EG, de Greeff SC, Dorigo-Zetsma JW (2003) A prospective, community-based study on virologic assessment among elderly people with and without symptoms of acute respiratory infection. J Clin Epidemiol 56:1218-1223

Holshue ML, DeBolt C, Lindquist S, Lofy KH, Wiesman J, Bruce H, Spitters C, Ericson K, Wilkerson S, Tural A, Diaz G (2020) First case of 2019 novel coronavirus in the United States. N Engl J Med 382:929-936. https://doi.org/10.1056/nejmoa2001191

Jin Z, Du X, Xu Y et al (2020) Structure of Mpro from COVID-19 virus and discovery of its inhibitors. Nature. https://doi.org/10.1038/ s41586-020-2223-y

Kim JM, Chung YS, Jo HJ, Lee NJ, Kim MS, Woo SH, Park S, Kim JW, Kim HM, Han MG (2020) Identification of coronavirus isolated from a patient in Korea with COVID-19. Osong Public Health Res Perspect 11:3-7

Komarnytsky S, Cook A, Raskin I (2011) Potato protease inhibitors inhibit food intake and increase circulating cholecystokinin levels by a trypsin-dependent mechanism. Int J Obes (Lond) 35(2):236243. https://doi.org/10.1038/ijo.2010.192

Lee PI, Hsueh PR (2020) Emerging threats from zoonotic coronaviruses-from SARS and MERS to 2019-nCoV. J Microbiol Immunol Infect 53:365-367

Lu H (2020) Drug treatment options for the 2019-new coronavirus (2019-nCoV). Biosci Trends 14(1):69-71. https://doi.org/10.5582/ bst.2020.01020

Mackay IM, Arden KE, Speicher DJ, O’Neil NT, McErlean PK, Greer RM, Nissen MD, Sloots TP (2012) Co-circulation of four human coronaviruses (HCoVs) in Queensland children with acute respiratory tract illnesses in 2004. Viruses 4:637-653

Pettersen EF, Goddard TD, Huang CC, Couch GS, Greenblatt DM, Meng EC, Ferrin TE (2004) UCSF Chimera: a visualization system for exploratory research and analysis. J Comput Chem 25(13):1605-1612. https://doi.org/10.1002/jcc.20084

Rodríguez-Morales AJ, MacGregor K, Kanagarajah S, Patel D, Schlagenhauf P (2020) Going global-travel and the 2019 novel coronavirus. Travel Med Infect Dis 33:10158. https://doi.org/10.1016/j. tmaid.2020.101578 
Vabret A, Mourez T, Gouarin S, Petitjean J, Freymuth F (2003) An outbreak of coronavirus OC43 respiratory infection in Normandy, France. Clin Infect Dis 36:985-989

Vabret A, Dina J, Gouarin S, Petitjean J, Tripey V, Brouard J, Freymuth F (2008) Human (non-severe acute respiratory syndrome) coronavirus infections in hospitalised children in France. J Paediatr Child Health 44:176-181

van Elden LJ, van Loon AM, van Alphen F, Hendriksen KA, Hoepelman AI, van Kraaij MG, Oosterheert JJ, Schipper P, Schuurman R, Nijhuis M (2004) Frequent detection of human coronaviruses in clinical specimens from patients with respiratory tract infection by use of a novel real-time reverse-transcriptase polymerase chain reaction. J Infect Dis 189:652-657
Zhijian Xu, Shi CPeng Y, Zhu Z, Mu K, Wang X, Zhu W (2020) Nelfinavir was predicted to be a potential inhibitor of 2019-nCov main protease by an integrative approach combining homology modelling, molecular docking and binding free energy calculation. bioRxiv. https://doi.org/10.1101/2020.01.27.921627

Zhou P, Yang X, Wang X et al (2020) A pneumonia outbreak associated with a new coronavirus of probable bat origin. Nature 579:270-273. https://doi.org/10.1038/s41586-020-2012-7

Publisher's Note Springer Nature remains neutral with regard to jurisdictional claims in published maps and institutional affiliations. 